\title{
New PDLI inhibitors for non-small cell lung cancer: focus on pembrolizumab
}

This article was published in the following Dove Press journal: OncoTargets and Therapy

\author{
Olivier Bylicki' \\ Nicolas Paleiron ${ }^{2}$ \\ Gaëlle Rousseau-Bussac ${ }^{3}$ \\ Christos Chouaïd ${ }^{3}$ \\ 'Pneumology Department, Hôpital \\ d'Instruction des Armées Percy, Paris, \\ ${ }^{2}$ Respiratory Department, Hôpital \\ d'Instruction des Armées Saint-Anne, \\ Toulon, ${ }^{3}$ Pneumology Department, \\ $\mathrm{CHI}$ Créteil, Paris, France
}

\begin{abstract}
The advent of immune-checkpoint inhibitors during the past decade represents a major advancement in the treatment of non-small cell lung cancer (NSCLC) with personalized treatment. Platinum-based chemotherapy has reached its efficacy threshold, with its use remaining limited by its toxicity. For NSCLC, inhibitors of the PD1 protein and its ligand PDL1 show promising clinical activity and induce durable responses in patients with advanced disease. The US Food and Drug Administration has approved pembrolizumab for treatment-naïve metastatic NSCLC with $\geq 50 \%$ of tumor cells expressing PDL1 and for metastatic NSCLC with $\geq 1 \%$ PDL1 expression after progression following first-line platinum-based doublet chemotherapy. In 2017, it also authorized the first-line combination of pembrolizumab and carboplatin-pemetrexed chemotherapy without selection based on PDL1 expression, but European health authorities are still waiting for the results of a Phase III trial. In this review, the clinical results of published and ongoing studies evaluating pembrolizumab for advanced NSCLC are analyzed and the potential role of PDL1 as a factor predictive of overall responses addressed.
\end{abstract}

Keywords: pembrolizumab, immune-checkpoint inhibitor, NSCLC, PDL1

\section{Introduction}

Lung cancer is a major public health problem, with an estimated 1.8 million new cases worldwide in 2012 , representing $12.9 \%$ of new cancers and $19.4 \%$ of cancer-attributable deaths. ${ }^{1,2}$ Non-small cell lung cancer (NSCLC) represents $85 \%$ of lung cancer. ${ }^{1,3}$ NSCLC prognosis is mediocre, with 5-year overall survival (OS) $<15 \%$, and until recently, therapeutic options beyond first-line treatment remained limited for patients. ${ }^{4-7}$

In the early 2000 s, the management of NSCLC patients was based on a combination of platinum and third-generation chemotherapy (pemetrexed, gemcitabine, and paclitaxel), with OS lasting about 12 months. The arrival of targeted therapies has transformed management for some patients $(\sim 15 \%)$, but the majority of patients cannot benefit from these treatments. The addition of bevacizumab to chemotherapy and maintenance strategies has resulted in a life gain of a few weeks for eligible patients. Despite these therapeutic advances, most patients with advanced or metastatic NSCLC received the same chemotherapy in 2015 as in 2005 . Results of several recent studies initially revealed the potential of second-line immune-checkpoint inhibitors (ICIs) for these patients and even as first-line therapy. ${ }^{8-13}$ Herein, we review clinical trial results and try to discern where pembrolizumab (Keytruda; Merck, Kenilworth, NJ, USA) fits best in the management of metastatic NSCLC.

\section{Immune checkpoints in cancer}

The immune system has long been considered a key actor in the surveillance and rejection of malignant tumors. ${ }^{14-16}$ Cancer cells generally have genetic and/or epigenetic

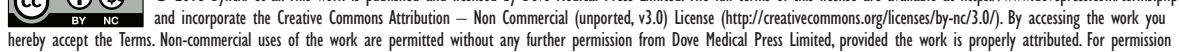
for commercial use of this work, please see paragraphs 4.2 and 5 of our Terms (https://www.dovepress.com/terms.php). 
alterations that can lead to the synthesis of neoantigens recognizable as "nonself" by the host's immune system. However, its responses can be limited by numerous immunosuppression mechanisms that render antitumor immunity ineffective. ${ }^{15}$ Diverse mechanisms have been described in the literature, notably negative regulation of antigen presentation; autoimmunization, which is T-lymphocyte recognition of specific tumor antigens that leads to the proliferation of clones deprived of immunodominant antigens; induction of self-tolerance, meaning that $\mathrm{T}$ lymphocytes are unable to kill tumor cells expressing the antigen; and finally, positive regulation of the immune control points (checkpoints) in the peritumoral microenvironment. ${ }^{17,18}$

ICIs are intended to block immune-system control points, with the goal of inducing the proliferation of immune cells and their activation against cancer cells. One of these checkpoints is the axis of the PD1 protein and its ligand PDL1. ${ }^{17} \mathrm{PD} 1$ is expressed at the surface of activated T cells, B lymphocytes, and natural killer cells. ${ }^{19}$ The interaction of PD1 with one of its two ligands, PDL1 or PDL2, engenders perturbed intracellular signaling and negative regulations of effector T-cell functions. ${ }^{20,21}$ PDL2 is expressed mainly on antigen-presenting cells, and PDL1 can be expressed on diverse cell types, including $\mathrm{T}$ lymphocytes, and epithelial and endothelial cells. PDL1 can also be expressed by tumor cells and other cells in the local tumor environment. ${ }^{17,22,23}$

PD1 is strongly expressed on the surface of activated $\mathrm{T}$ lymphocytes in response to inflammation or infection. Tumor cells can escape the immunoresponse via regulated PDL1 expression. ${ }^{17}$ When PD1 is bound to its ligand PDL1, the resulting complex blocks the immunoresponse by inhibiting the cytotoxic T-lymphocyte response. ICIs interrupt that, inhibiting T-cell signaling, thereby reactivating the antitumor actions of specific cytotoxic T cells. ${ }^{17,24,25}$ NSCLC cells frequently express PDL1, with up to $68 \%$ of tumors being $\mathrm{PDL}^{+}{ }^{+}$according to the literature. ${ }^{9,26}$

\section{Pembrolizumab}

Pembrolizumab (Keytruda, MK3475), humanized immunoglobulin $G_{4}$, was approved to treat refractory melanoma in 2015 by the US Food and Drug Administration (FDA) and in Europe by the European Medicines Agency (EMA). ${ }^{27}$ That year, the FDA and EMA also approved pembrolizumab to treat metastatic NSCLC expressing PDL1 $(>1 \%)$ and progressing after platinum-based chemotherapy (or after chemotherapy and tyrosine-kinase inhibitors targeting EGFR mutations or ALK rearrangement). ${ }^{6,13}$ More recently, pembrolizumab was approved by the FDA, EMA, and the
Japanese Pharmacological and Medical Devices Agency to treat patients with chemotherapy-naïve, metastatic NSCLC expressing high PDL1 ( $\geq 50 \%$ ) levels without $E G F R$ mutation or $A L K$ rearrangement. Finally, in January 2017, the FDA accelerated approval of the combination of pembrolizumab and pemetrexed-carboplatin chemotherapy (based on KEYNOTE-021 results) as first-line treatment for metastatic or advanced nonsquamous-cell NSCLC without EGFR mutation or ALK rearrangement, regardless of PDL1 expression.

\section{Second-line pembrolizumab}

Pembrolizumab's clinical activity and safety were evaluated in the international multicenter Phase I KEYNOTE-001 trial (NCT01295827) of 495 untreated (101 first-line therapy) or previously treated (394 second-line or more) patients. ${ }^{28}$ All patients received pembrolizumab intravenously (IV): 2 or $10 \mathrm{mg} / \mathrm{kg}$ every 3 weeks (Q3W) or $10 \mathrm{mg} / \mathrm{kg}$ Q2W. Primary end points were safety and efficacy: objective response rate (ORR), OS, progression-free survival (PFS), and duration of response (DOR). ${ }^{28}$ Observation during the trial of an association between tumor-cell PDL1 expression and pembrolizumab effectiveness led to a protocol amendment, with the adjunction of another principal outcome criterion: efficacy in patients with high PDL1-expressing tumors. ${ }^{29}$

In that study, the safety profile of pembrolizumab was acceptable. All-grade treatment-associated adverse events (AEs) combined occurred in $71 \%$ of the patients, without any difference among doses given or frequency; grade $\geq 3$ AEs were reported in $10 \%$ of patients. ORR for the entire population was $19 \%$ (95\% CI 16\%-23\%), with $22 \%$ of patients with stable disease. ORR for previously treated patients was $18 \%$ (95\% CI 14\%-22\%). Median PFS and OS, respectively, were 3.7 (95\% CI 2.9-4.1) and 12.0 (95\% CI 9.3-14.7) months at the time of analysis.

After median follow-up of 22 months, median OS rates for treatment-naïve and previously treated patients, respectively, were 22.1 (95\% CI 16.8-27.2) and 10.6 (95\% CI 8.6-13.3) months. ${ }^{30}$ ORR, 12-month PFS, and 12-month OS, respectively, were $51.9 \%, 54 \%$, and $85 \%$ for patients with $\geq 50 \%$ PDL1-expressing tumor cells compared to $26.7 \%, 35 \%$, and $71 \%$ for the overall population. Those updated results confirmed pembrolizumab safety, with only 12 (11.9\%) patients experiencing treatment-associated grade 3/4 AEs and no deaths. ${ }^{28,30,31}$ Those findings, promising in terms of safety and efficacy, led to several Phase I-III trials (Table 1) to evaluate pembrolizumab alone or in combination with other treatments for patients with advanced NSCLC. ${ }^{10-12}$ 
Table I Main results of the seven trials that evaluated first- and second-line pembrolizumab for non-small cell lung cancer

\begin{tabular}{|c|c|c|c|c|c|c|c|c|c|c|}
\hline & $\mathbf{n}$ & Treatment arm & $\mathbf{n}$ & $\begin{array}{l}\text { OS, } \\
\text { months }\end{array}$ & HR (95\% Cl) & $P$-value & PFS & HR (95\% CI) & $P$-value & ORR (\%) \\
\hline \multicolumn{11}{|c|}{ KEYNOTE-00 I Phase $\left.\right|^{28}$} \\
\hline \multirow[t]{3}{*}{ PDLI $>1 \%$} & \multirow[t]{3}{*}{495} & $\begin{array}{l}\text { Pembrolizumab } \\
2 \mathrm{mg} / \mathrm{kg} \text { Q3W }\end{array}$ & 6 & & - & & & - & & 19.4 \\
\hline & & $\begin{array}{l}\text { Pembrolizumab } \\
10 \mathrm{mg} / \mathrm{kg} \text { Q3W }\end{array}$ & 287 & \multirow[t]{2}{*}{12} & - & & \multirow[t]{2}{*}{3.7} & - & & \\
\hline & & $\begin{array}{l}\text { Pembrolizumab } \\
10 \mathrm{mg} / \mathrm{kg} \text { Q2W }\end{array}$ & 202 & & - & & & - & & \\
\hline \multicolumn{11}{|c|}{ KEYNOTE-0IO Phase II/III'0 } \\
\hline \multirow[t]{3}{*}{$\mathrm{PDLI}>1 \%$} & \multirow[t]{3}{*}{$\mathrm{I}, 034$} & $\begin{array}{l}\text { Pembrolizumab } \\
2 \mathrm{mg} / \mathrm{kg} \text { Q3W }\end{array}$ & 345 & 10.4 & $\begin{array}{l}0.71 \\
(0.58-0.88)\end{array}$ & 0.008 & 3.9 & $\begin{array}{l}0.88 \\
(0.74-1.05)\end{array}$ & 0.07 & 18 \\
\hline & & $\begin{array}{l}\text { Pembrolizumab } \\
10 \mathrm{mg} / \mathrm{kg} \text { Q3W }\end{array}$ & 346 & 12.7 & $\begin{array}{l}0.61 \\
(0.49-0.75)\end{array}$ & 0.001 & 4 & $\begin{array}{l}0.79 \\
(0.66-0.94)\end{array}$ & 0.004 & 18.5 \\
\hline & & Docetaxel & 343 & 8.5 & - & & 4 & - & & 9.3 \\
\hline \multicolumn{11}{|c|}{ KEYNOTE-024 Phase III" } \\
\hline \multirow[t]{2}{*}{ PDLI >50\% } & \multirow[t]{2}{*}{305} & $\begin{array}{l}\text { Pembrolizumab } 200 \text { mg } \\
\text { Q3W }\end{array}$ & 154 & NR & $\begin{array}{l}0.60 \\
(0.41-0.89)\end{array}$ & 0.005 & 10.3 & $\begin{array}{l}0.50 \\
(0.37-0.68)\end{array}$ & 0.001 & 44.8 \\
\hline & & $\begin{array}{l}\text { Platinum-based } \\
\text { chemotherapy }\end{array}$ & $|5|$ & NR & & & 6 & & & 27.8 \\
\hline \multicolumn{11}{|c|}{ KEYNOTE-02 I Phase II/III'2 } \\
\hline \multirow[t]{2}{*}{ PDLI not selected } & \multirow[t]{2}{*}{123} & $\begin{array}{l}\text { Pembrolizumab + } \\
\text { carboplatin-pemetrexed }\end{array}$ & 60 & - & - & & 13 & $\begin{array}{l}0.50 \\
(0.31-0.91)\end{array}$ & 0.01 & 55 \\
\hline & & Carboplatin-pemetrexed & 63 & - & - & & 8.9 & & & 29 \\
\hline \multicolumn{11}{|c|}{ KEYNOTE-I 89 Phase III $^{36}$} \\
\hline \multirow[t]{2}{*}{ PDLI not selected } & \multirow[t]{2}{*}{616} & $\begin{array}{l}\text { Pembrolizumab + } \\
\text { carboplatin-pemetrexed }\end{array}$ & 410 & NR & $\begin{array}{l}0.49 \\
(0.38-0.64)\end{array}$ & 0.001 & 8.8 & $\begin{array}{l}0.52 \\
(0.43-0.64)\end{array}$ & 0.001 & 47.6 \\
\hline & & Carboplatin-pemetrexed & 206 & & & & & & & 18.9 \\
\hline \multicolumn{11}{|c|}{ KEYNOTE-042 Phase III ${ }^{38}$} \\
\hline \multirow[t]{2}{*}{ PDLI $>1 \%$} & \multirow[t]{2}{*}{1,274} & $\begin{array}{l}\text { Pembrolizumab + } \\
\text { platinum-based } \\
\text { chemotherapy }\end{array}$ & 637 & 16.7 & $\begin{array}{l}0.8 \mathrm{I} \\
(0.7 \mathrm{I}-0.93)\end{array}$ & 0.0018 & 5.4 & $\begin{array}{l}1.07 \\
(0.94-1.21)\end{array}$ & & 27.3 \\
\hline & & $\begin{array}{l}\text { Platinum-based } \\
\text { chemotherapy }\end{array}$ & 637 & 12.1 & - & & 6.5 & & & 26.5 \\
\hline \multicolumn{11}{|c|}{ KEYNOTE-407 Phase III $^{39}$} \\
\hline \multirow[t]{2}{*}{ PDLI not selected } & \multirow[t]{2}{*}{559} & $\begin{array}{l}\text { Pembrolizumab }+ \\
\text { carboplatin-paclitaxel or } \\
\text { nab-paclitaxel }\end{array}$ & 278 & 15.9 & $\begin{array}{l}0.63 \\
(0.49-0.85)\end{array}$ & 0.0008 & 6.4 & $\begin{array}{l}0.56 \\
(0.45-0.70)\end{array}$ & 0.0001 & 58.4 \\
\hline & & $\begin{array}{l}\text { Carboplatin-paclitaxel or } \\
\text { nab-paclitaxel }\end{array}$ & 281 & 11.3 & - & & 4.8 & & & 35.0 \\
\hline
\end{tabular}

Abbreviations: HR, hazard ratio; OS, overall survival; PFS, progression-free survival; ORR, objective response rate; Q3W, every 3 weeks; NR, not reported.

Pembrolizumab was approved as second-line therapy for NSCLC based on the results of the open Phase II-III randomized KEYNOTE-010 trial, ${ }^{10}$ which evaluated its efficacy and safety vs docetaxel for patients with advanced NSCLC whose cells expressed low PDL1 ( $\geq 1 \%$ ) levels. Patients were randomized to receive one of three treatments Q3W: pembrolizumab $2(\mathrm{n}=345)$ or $10 \mathrm{mg} / \mathrm{kg}(\mathrm{n}=346)$, or docetaxel $75 \mathrm{mg} / \mathrm{m}^{2}(\mathrm{n}=343)$. Primary end points were OS and PFS for all patients and the subgroup with tumors expressing $\geq 50 \%$ PDL1. Using PDL1 expression as a companion test, and its pertinence, will be discussed in the section on biomarkers. Secondary end points included tolerance, ORR, and DOR. The trial included 1,034 patients. Compared to docetaxel, the 2 and $10 \mathrm{mg} / \mathrm{kg}$ pembrolizumab doses, respectively, prolonged OS - 10.4 (95\% CI 9.4-11.9), 12.7 (95\% CI 10.0-17.3) vs 8.5 (95\% CI 7.5-9.8) months with no difference between the two pembrolizumab doses (hazard ratio [HR] 1.17, 95\% CI 0.94-1.45), but PFS rates 
did not differ significantly. After subgroup analyses, no significant OS benefit was observed with pembrolizumab compared to docetaxel for women (HR 1.02, 95\% CI 0.781.32), patients with EGFR-mutated tumors (HR 1.79, 95\% CI 0.94-3.42), or those whose tumors had 1\%-49\% PDL1 expression (HR 1.04, 95\% CI 0.85-1.27).

Pembrolizumab efficacy against tumors with $\geq 50 \%$ PDL1 expression was a principal end-point cocriterion. Among the 2,222 patients screened, 1,475 (66\%) had $\geq 1 \%$ PDL1 expression, with 633 (28\%) having $\geq 50 \%$ PDL1 and thus were assessable for the cocriterion. Those patients' median OS rates for the 2 and $10 \mathrm{mg} / \mathrm{kg}$ pembrolizumab and docetaxel groups, respectively, were 14.9 (95\% CI 10.4-not reached), 17.3 (11.8-not reached), and 8.2 (6.4-10.7) months. OS was comparable for the two pembrolizumab arms. Pembrolizumab's safety profile was better than that of docetaxel, with fewer grade 3/4 AEs, in agreement with Phase I trial results. ${ }^{28}$ Grade $\geq 3$ AEs were reported in 13\% (43 of 339), $16 \%$ (55 of 343), and 35\% (109 of 309) of patients given 2 or $10 \mathrm{mg} / \mathrm{kg}$ pembrolizumab or docetaxel, respectively. Among the eleven treatment-attributed deaths, three occurred in the $2 \mathrm{mg} / \mathrm{kg}$ pembrolizumab arm (two interstitial and one infectious pneumopathy), three in $10 \mathrm{mg} / \mathrm{kg}$ pembrolizumab recipients (one myocardial infarction and one interstitial or one infectious pneumopathy), and five in the docetaxel group. Immune-related AEs (IRAEs), regardless of grade, occurred in 20\% (69 of 339) and 19\% (64 of 343) of patients who received 2- or $10 \mathrm{mg}$ of $\mathrm{kg}$ pembrolizumab, respectively. The most common IRAEs were hypothyroidism, hyperthyroidism and interstitial pneumopathy.

\section{First-line pembrolizumab}

Several clinical trials have evaluated first-line pembrolizumab. An analysis of 101 treatment-naïve patients included in the Phase-1 (KEYNOTE-001) trial was published in 2017. ${ }^{30}$ ORR (51.9\%), 12-month PFS (54\%), and 12-month OS (84\%) for patients with PDL1 $\geq 50 \%$ NSCLC were better than for the entire population $(26.7 \%, 35 \%$, and $71 \%$, respectively). Those findings led to Phase I-III trials testing the molecule as first-line therapy.

The Phase III randomized KEYNOTE-024 trial (NCT02142738) compared pembrolizumab (200 mg IV, 3QW) until disease progression or unacceptable toxicity for a maximum of 2 years vs standard platinum-based doublet chemotherapy (four to six cycles with maintenance therapy possible) as first-line therapy for advanced NSCLC without EGFR mutation or $A L K$ rearrangement, but with PDL1 $\geq 50 \%$ determined by immunohistochemistry (IHC). ${ }^{11}$ The primary end point was PFS and secondary-outcome criteria were
ORR, OS, and safety. Patients with untreated brain metastases, active autoimmune disease requiring systemic therapy, or performance status (PS) $\geq 1$ were excluded. Patients who received platinum-based chemotherapy (cisplatin $75 \mathrm{mg} / \mathrm{m}^{2}$ IV or carboplatin area under the carboplatin area under curve (AUC) 5-6 IV Q3W) in combination with pemetrexed (500 mg/m² IV Q3W), gemcitabine (1,250 mg/m² IV Q3W), or paclitaxel (200 mg/m² IV Q3W with carboplatin) served as controls. Among 1,934 patient tumors screened for PDL1, $500(25.9 \%)$ showed PDL $1 \geq 50 \%$. Finally, 305 patients were included and randomized (154 to the pembrolizumab arm and 151 to the control group). ${ }^{11}$ In the case of disease progression in the control group, crossover was authorized.

At the time of analysis, $50 \%$ of chemotherapy-treated controls had switched to pembrolizumab because of progression vs $23 \%$ of the pembrolizumab-arm patients. The main results are summarized in Table 1. Median PFS rates were 10.4 vs 6.0 months for the pembrolizumab and chemotherapy arms, respectively (HR 0.5, 95\% CI 0.37-0.68; $P<0.001)$. OS was also significantly better for patients in the pembrolizumab arm, with the median not reached for either arm. Estimated OS rates at 6 months were $80.2 \%$ and $72.4 \%$, respectively, for the pembrolizumab and control arms (HR $0.60,95 \%$ CI $0.41-0.89 ; P=0.005)$, with respective ORRs of $45 \%$ and $28 \%(P=0.0011)$. A PFS benefit was observed for all the predefined subgroups.

The data were updated at the 2017 World Conference on Lung Cancer. ${ }^{32}$ At data cutoff (July 10, 2017) after a median follow-up of 25.2 months, the HR for OS was 0.63 (95\% CI $0.47-0.86, P=0.002$ ). Median OS was 30.0 (18.3-not reached) months for the pembrolizumab arm and 14.2 (9.8-19.0) months for the chemotherapy arm. Those groups' respective 12-month OS rates were 70.3\% (95\% CI $62.3 \%-76.9 \%)$ vs $54.8 \%$ (95\% CI 46.4\%-62.4\%). ${ }^{32}$

All-grade AEs combined occurred in $73.4 \%$ and $90.0 \%$ of patients in the pembrolizumab and control groups, respectively. ${ }^{11}$ Grade 3/4 AEs were observed in 26\% of pembrolizumab recipients and $51 \%$ of chemotherapy-treated controls. The main grade 3/4 non-IRAEs associated with pembrolizumab were diarrhea (3.9\%), anemia (1.9\%), and fatigue $(1.3 \%)$. The overall IRAE frequency under pembrolizumab was $29.2 \%$, among which $9.7 \%$ was grade $\geq 3$, mainly pulmonary $(2.6 \%)$, cutaneous $(3.9 \%)$, and digestive $(1.3 \%)$.

In that trial, ${ }^{33}$ patients' quality of life was evaluated with the Quality of Life Questionnaire - cancer (QLQ-C)-30 and QLQ - lung cancer (QLQ LC)-13. Mean variations in QLQ-C30 scores compared to their initial values were 6.9 (95\% CI 0.3-10.6) points for pembrolizumab and -0.9 (95\% CI -4.8 to 3.0 ) for the controls, for a difference of 
7.8 (95\% CI 2.9-12.8, $P=0.002$ ). Fewer pembrolizumabtreated patients than chemotherapy controls $(31 \%$ vs $39 \%$, respectively) had poorer QLQ-LC13 composite scores, with longer time to deterioration under pembrolizumab than chemotherapy (HR $0.66,95 \%$ CI $0.44-0.97 ; P=0.029$ ). ${ }^{33}$ These findings led to premature termination of the trial by the independent monitoring committee, with a recommendation to offer chemotherapy-treated patients the option to receive pembrolizumab. ${ }^{11}$ Based on these results, the FDA, EMA, and Japanese Pharmacological and Medical Devices Agency approved pembrolizumab for first-line treatment of metastatic NSCLC with elevated PDL1 ( $\geq 50 \%$ ) expression.

\section{Ongoing trials of pembrolizumab in combination}

Pembrolizumab was also evaluated in combination with chemotherapy as first-line NSCLC treatment, regardless of PDL1 expression, in a Phase I/II trial (KEYNOTE-021) comprised of eight cohorts: ${ }^{12,34}$ cohort A, pembrolizumab $2 / 10 \mathrm{mg} / \mathrm{kg}+$ carboplatin $\mathrm{AUC}_{6}+$ paclitaxel $200 \mathrm{mg} / \mathrm{m}^{2}$; cohort B, pembrolizumab 2/10 mg/kg + carboplatin $\mathrm{AUC}_{6}+$ paclitaxel $200 \mathrm{mg} / \mathrm{m}^{2}+$ bevacizumab $15 \mathrm{mg} / \mathrm{kg}$; cohort $\mathrm{C}$, pembrolizumab $2 / 10 \mathrm{mg} / \mathrm{kg}+$ carboplatin $\mathrm{AUC}_{5}+$ pemetrexed $500 \mathrm{mg} / \mathrm{m}^{2}$; cohort D, pembrolizumab $2 \mathrm{mg} / \mathrm{kg}+$ ipilimumab $1 \mathrm{mg} / \mathrm{kg}$; cohort E, pembrolizumab $2 \mathrm{mg} / \mathrm{kg}+$ erlotinib $150 \mathrm{mg}$; cohort F, pembrolizumab $2 \mathrm{mg} / \mathrm{kg}$ + gefitinib $250 \mathrm{mg}$; cohort $\mathrm{G}$, carboplatin $\mathrm{AUC}_{5}+$ pemetrexed $500 \mathrm{mg} / \mathrm{m}^{2}$ with/without pembrolizumab $200 \mathrm{mg}$; and cohort $\mathrm{H}$, extension of cohort $\mathrm{D}$.

KEYNOTE-021 results are available only for cohorts A, $\mathrm{B}, \mathrm{D}, \mathrm{G}$, and $\mathrm{H}$, but not for cohorts $\mathrm{C}$, D, or E. KEYNOTE021 cohort $\mathrm{G}$ had the strongest clinical impact: the combination of pembrolizumab (200 mg IV) with pemetrexed $\left(500 \mathrm{mg} / \mathrm{m}^{2} \mathrm{IV}\right)$-carboplatin (PPC; $\mathrm{AUC}_{5}$ ) Q3W achieved a better ORR than chemotherapy alone. ${ }^{12}$ Patients with unmutated EGFR or unrearranged ALK NSCLC were treatmentnaïve and PS 0-1, without brain metastases or interstitial lung disease, and only systemic corticosteroid doses $<10 \mathrm{mg}$ /day were allowed. The primary end point was ORR, and secondary end points were PFS, OS, and pembrolizumab safety and efficacy according to PDL1 status. The 123 enrolled patients were randomized to the PPC $\operatorname{arm}(\mathrm{n}=60)$ or to receive pemetrexed-carboplatin (PC) chemotherapy alone $(n=63)$. A $26 \%$ ORR benefit was observed with pembrolizumab (ORR 55\% with PPC vs $29 \%$ with PC, $P=0.0016$ ), with respective median PFS of 13.0 vs 8.9 months (HR $0.53,95 \%$ CI $0.31-0.91 ; P=0.0102)$. OS did not differ between the two arms (HR 0.90, 95\% CI 0.42-1.91), with 1-year OS at 75\% and $72 \%$ for the PPC and PC arms, respectively. No effect of PDL1 status at the $<1 \%$ threshold was observed. With the $50 \%$ threshold, ORR differed: $26 \%$ for patients with $1 \%-49 \%$ PDL1 ( $n=19$ ) and $80 \%$ for the subgroup with $\geq 50 \%$ PDL1 expression $(\mathrm{n}=20)$.

All-grade AEs occurred in $93 \%$ of the PPC recipients and $90 \%$ of those given PC, among which $39 \%$ and $26 \%$, respectively, were grade $>2 .{ }^{12}$ The main PPC-associated AEs reported were fatigue (64\%), nausea (58\%), anemia (32\%), cutaneous eruptions (27\%), and vomiting (27\%). IRAEs in the pembrolizumab-treated group were hypothyroidism (15\%), hyperthyroidism (8\%), and interstitial pneumopathy (5\%). Those results, submitted to the FDA in January 2017, led to the approval of pembrolizumab in combination with (pemetrexed-carboplatin) chemotherapy for first-line treatment of advanced, nonsquamous-cell NSCLC without EGFR mutations or $A L K$ rearrangements, regardless of PDL1 status.

Updated data were presented at the 2018 American Society of Clinical Oncology (ASCO) Annual Meeting, with median follow-up at 24 months (range 0.8-29.0 months). ${ }^{35}$ ORR was $57 \%$ with PPC vs $30 \%$ for PC $(P=0.0016)$. PFS was significantly improved with PPC (24.0 months) vs PC (9.3 months; HR $0.53,95 \%$ CI $0.33-0.86 ; P=0.0049$ ). Median OS was not reached for PPC or PC, respectively: (24.5-not recorded [NR]) and $21.1(14.9-\mathrm{NR})$ months (HR 0.56, 95\% CI $0.32-0.95 ; P=0.0151)$. Twenty-four-month OS rates were $67 \%$ for the pembrolizumab arm and $48 \%$ for the placebo arm. ${ }^{35}$ These updated data confirmed the first published results that had led to FDA approval.

The Phase III KEYNOTE-189 trial included 616 patients (randomized 2:1) to confirm the results of the KEYNOTE021 trial on the efficacy of the pembrolizumab-pemetrexedplatinum combination for chemotherapy-naïve NSCLC without EGFR mutation or $A L K$ rearrangement (Table 1). ${ }^{36}$ The main end points were PFS and OS. The recently published results supported the use of that combination. PFS was prolonged by 3.9 months for the pembrolizumab arm (median PFS 8.8 months) compared to the placebo arm (median PFS 4.9 months) with HR 0.52 (95\% CI $0.43-0.64, P<0.001$ ), with respective 1-year OS rates of $69.5 \%$ and $49.4 \%$. According to subgroup analyses, no PFS benefit was obtained for PDL $1<1 \%$ (HR $0.75,95 \%$ CI $0.53-1.05$ ). Those findings stand out, regardless of tumor PDL1 status. After centralized review, ORRs were $46.7 \%$ for pembrolizumab recipients vs $18.9 \%$ for those given placebo $(P<0.001){ }^{36}$

During the trial, $41 \%$ of placebo arm patients crossed over to receive pembrolizumab monotherapy or other immunotherapies after progression. Grade $\geq 3$ toxicity rates for the pembrolizumab and placebo arms, respectively, were $67.3 \%$ and $65.2 \%$, with respective $20.2 \%$ and $10.7 \% \mathrm{AE}$ 
rates leading to discontinuation, and $8.9 \%$ and $4.5 \%$ grade $\geq 3$ IRAE rates. The most frequent secondary grade $\geq 3$ IRAEs were interstitial pneumopathy $(2.7 \%$, with three deaths), cutaneous reactions ( $2 \%$ ), and nephritides $(1.5 \%) .{ }^{36}$

ForKEYNOTE-021 cohortA(pembrolizumab 2/10 mg/kg+ carboplatin $\mathrm{AUC}_{6}+$ paclitaxel $200 \mathrm{mg} / \mathrm{m}^{2}$ ) Q3W, four cycles followed by pembrolizumab Q3W up to 2 years or progression, 13 of $25(52 \%)$ patients achieved partial responses. For cohort B (pembrolizumab 2/10 mg/kg + carboplatin $\mathrm{AUC}_{6}+$ paclitaxel $200 \mathrm{mg} / \mathrm{m}^{2}+$ bevacizumab $15 \mathrm{mg} / \mathrm{kg} \mathrm{Q} 3 \mathrm{~W}$ ), four cycles also obtained partial responses. During the follow-up of one arm by pembrolizumab-bevacizumab maintenance, 12 of 25 (48\%) patients had partial responses.
The pembrolizumab-ipilimumab combination given to KEYNOTE-021 cohorts D (pembrolizumab $2 \mathrm{mg} / \mathrm{kg}$, ipilimumab $1 \mathrm{mg} / \mathrm{kg}$ ) and $\mathrm{H}$ (cohort extension) led to $25 \%$ ORRs for 45 of the assessable patients given the pembrolizumab (2 mg/kg)-ipilimumab (1 mg/kg) combination, a rate similar to that obtained with pembrolizumab alone, and a $64 \%$ disease-control rate. ${ }^{37}$ The response was not associated with PDL1 status. Compared to immunotherapy alone, the combination's safety was not as good, with $49 \%$ of patients experiencing grade 3/4 AEs, necessitating treatment discontinuation for 9\%. All-grade IRAEs occurred in $40 \%$ of the patients. Principal ongoing trials are summarized in Table 2.

Table 2 Main ongoing trials evaluating pembrolizumab for non-small cell lung cancer

\begin{tabular}{|c|c|c|c|c|c|c|}
\hline Stage & $\begin{array}{l}\text { Specific } \\
\text { population }\end{array}$ & Phase & $\mathbf{n}$ & Experimental arm & Acronym & ClinicalTrials.gov \\
\hline \multicolumn{7}{|l|}{ Stage I } \\
\hline & & I & 20 & SBRT + pembrolizumab & & NCT034469II \\
\hline Neoadjuvant & & I & 28 & Pembrolizumab & MK3475-223 & NCT02938624 \\
\hline \multicolumn{7}{|l|}{ Stage II } \\
\hline Neoadjuvant & Squamous cell & II & 32 & Pembrolizumab & TOP I50I & NCT028I8920 \\
\hline Neoadjuvant & & II & 30 & Pembrolizumab & NEOMUN & NCT03।97467 \\
\hline Adjuvant & & III & I,38I & Pembrolizumab & $\begin{array}{l}\text { PEARLS or } \\
\text { KEYNOTE-092 }\end{array}$ & NCT02504373 \\
\hline \multirow[t]{2}{*}{ Adjuvant } & & $\mathrm{I} / \mathrm{II}$ & 202 & Pembrolizumab + entinostat & & NCT02437I36 \\
\hline & & 1 & 25 & Radiotherapy + pembrolizumab & & NCT03245I77 \\
\hline \multicolumn{7}{|l|}{ Stage III } \\
\hline Adjuvant & Stage IIIA (N2) & II & 37 & Pembrolizumab & & NCT03053856 \\
\hline $\begin{array}{l}\text { Neoadjuvant }+ \\
\text { consolidation }\end{array}$ & & I & 20 & $\begin{array}{l}\text { Neoadjuvant chemoradiation }+ \\
\text { pembrolizumab followed by pembrolizumab } \\
\text { consolidation }\end{array}$ & & NCT02987998 \\
\hline \multirow[t]{4}{*}{$\begin{array}{l}\text { Neoadjuvant or } \\
\text { adjuvant }\end{array}$} & $\| \mathrm{IB} / \mathrm{II} \mathrm{A}$ & III & 786 & $\begin{array}{l}\text { Pembrolizumab + platinum-doublet } \\
\text { chemotherapy }\end{array}$ & KEYNOTE-67I & NCT03425643 \\
\hline & IIB/IIIB & I & 30 & $\begin{array}{l}\text { Pembrolizumab }+ \text { paclitaxel }+ \text { carboplatin }+ \\
\text { radiation therapy }\end{array}$ & & NCT0262।398 \\
\hline & $\begin{array}{l}\text { Unresectable } \\
\text { stage III }\end{array}$ & II & 126 & Pembrolizumab maintenance & MP-LALC & NCT0337944I \\
\hline & $\begin{array}{l}\text { Unresectable } \\
\text { stage III }\end{array}$ & II & 95 & $\begin{array}{l}\text { Pembrolizumab consolidation following } \\
\text { chemoradiation }\end{array}$ & & NCT02343952 \\
\hline \multirow[t]{2}{*}{ Adjuvant } & $\begin{array}{l}\text { Stage III with } \\
\text { EGFR mutation }\end{array}$ & II & 25 & Pembrolizumab & & NCT02879994 \\
\hline & Advanced & I & 37 & Pembrolizumab & KEYNOTE-025 & NCT02007070 \\
\hline \multirow[t]{2}{*}{ Adjuvant } & & II & 75 & Pembrolizumab after SBRT & & NCT02492568 \\
\hline & $\begin{array}{l}\text { Localized } \\
\text { recurrence }\end{array}$ & II & 41 & $\begin{array}{l}\text { Pembrolizumab consolidation after concurrent } \\
\text { chemotherapy and proton reirradiation }\end{array}$ & & NCT03087760 \\
\hline \multicolumn{7}{|l|}{ Stage IV } \\
\hline First line & $\geq 50 \%$ PDLI & III & 548 & Pembrolizumab + ipilimumab & KEYNOTE-598 & NCT03302234 \\
\hline First line & & II & 20 & Pembrolizumab + carboplatin + paclitaxel & & NCT02581943 \\
\hline
\end{tabular}


Table 2 (Continued)

\begin{tabular}{|c|c|c|c|c|c|c|}
\hline Stage & \begin{tabular}{|l|} 
Specific \\
population
\end{tabular} & Phase & n & Experimental arm & Acronym & ClinicalTrials.gov \\
\hline First line & & II & 90 & GRN-I20I + pembrolizumab & & NCT034I 7882 \\
\hline First line & & II & 65 & Pembrolizumab for PDLI-low tumors & PEOPLE & NCT03447678 \\
\hline First line & & II & 90 & $\begin{array}{l}\text { Optimal sequencing of pembrolizumab }+ \\
\text { standard platinum-based chemotherapy }\end{array}$ & & NCT0259I6I5 \\
\hline First line & & II & 100 & Pembrolizumab + AM00I0 & Cypress I & NCT03382899 \\
\hline First line & & I/II & 44 & $\begin{array}{l}\text { Pembrolizumab + oral decitabine and } \\
\text { tetrahydrouridine }\end{array}$ & & NCT03233724 \\
\hline First line & & II & 26 & AGENI884 + pembrolizumab & & NCT034II473 \\
\hline $\begin{array}{l}\text { Maintenance after } \\
\text { first line }\end{array}$ & & II & 48 & Switch to pembrolizumab maintenance & SWIPE & NCT02705820 \\
\hline $\begin{array}{l}\text { Maintenance after } \\
\text { first line }\end{array}$ & Squamous cell & II & 130 & $\begin{array}{l}\text { Pembrolizumab maintenance after platinum- } \\
\text { based chemotherapy }\end{array}$ & PRIMUS & NCT02564380 \\
\hline Second line & I\%-49\% PDLI & III & 740 & Pembrolizumab & & NCT02864394 \\
\hline Second line or more & & 1 & 84 & Pembrolizumab & KEYNOTE OII & NCT0I 840579 \\
\hline Second line or more & & II & 105 & $\begin{array}{l}\text { Sequential consolidation: pembrolizumab then } \\
\text { nab-paclitaxel }\end{array}$ & & NCT0268446I \\
\hline Second line or more & & I/II & 508 & Pembrolizumab + epacadostat & KEYNOTE-037 & NCT02I78722 \\
\hline Second line or more & & III & 588 & Pembrolizumab + epacadostat & $\begin{array}{l}\text { KEYNOTE } \\
654-01\end{array}$ & NCT03322540 \\
\hline Second line or more & & I/II & 421 & $\begin{array}{l}\text { Pembrolizumab + epacadostat }+ \\
\text { chemotherapy }\end{array}$ & KEYNOTE-723 & NCT030859 I 4 \\
\hline Second line or more & & III & 1,062 & $\begin{array}{l}\text { Pembrolizumab + epacadostat } \pm \text { platinum- } \\
\text { based chemotherapy }\end{array}$ & $\begin{array}{l}\text { KEYNOTE- } \\
715-02\end{array}$ & NCT03322566 \\
\hline Second line or more & & I/II & 200 & Pembrolizumab + CV30I & $\begin{array}{l}\text { MAGNI- } \\
\text { LUNG-0I }\end{array}$ & NCT02840994 \\
\hline Second line or more & & I/II & 46 & Pembrolizumab + gemcitabine & & NCT0242238I \\
\hline Second line or more & & 1 & 90 & Pembrolizumab + CVA2I & STROM & NCT02043665 \\
\hline Second line or more & & 1 & 81 & Pembrolizumab + PEGPH20 & & NCT02563548 \\
\hline Second line or more & & 1 & 75 & Pembrolizumab + necitumumab & & NCT0245I930 \\
\hline Second line or more & & 1 & 20 & $\begin{array}{l}\text { Personalized therapeutic antitumor vaccine }+ \\
\text { pembrolizumab }\end{array}$ & & NCT03I 66254 \\
\hline Second line or more & & 1 & 40 & CVA2I + pembrolizumab & & NCT02824965 \\
\hline Second line or more & & I/II & 36 & Pembrolizumab + Imprime PGG & & NCT03003468 \\
\hline Second line or more & & II & 105 & Pembrolizumab + ALT803 & & NCT03228667 \\
\hline Second line or more & & 1 & 36 & Pembrolizumab + nab-paclitaxel & $\begin{array}{l}\text { URCOH-PMS- } \\
001\end{array}$ & NCT02733250 \\
\hline Second line or more & & II & 70 & Pembrolizumab + docetaxel & & NCT02574598 \\
\hline Second line or more & & I/II & 44 & Pembrolizumab + lenalidomide & & NCT029636I0 \\
\hline Second line or more & & $\mid / / I$ & 61 & $\begin{array}{l}\text { Pembrolizumab + oncolytic MG I-MAGEA3 } \\
\text { with Ad-MAGEA3 vaccine }\end{array}$ & & NCT02879760 \\
\hline Second line or more & & II & 48 & Pembrolizumab + itacitinib & & NCT03425006 \\
\hline Second line or more & & 1 & 237 & $\begin{array}{l}\text { Pembrolizumab + itacitinib or pembrolizumab } \\
+ \text { NCB050465 }\end{array}$ & & NCT02646748 \\
\hline Second line or more & Adenocarcinoma & II & 48 & Pembrolizumab + BGB324 & & NCT03 I8457I \\
\hline Second line or more & & 1 & 75 & Pembrolizumab + enoblituzumab (MGA27I) & & NCT024752I3 \\
\hline Second line or more & & I/II & 100 & Pembrolizumab + vorinostat & & NCT02638090 \\
\hline Second line or more & & $\mid / / I$ & 142 & Pembrolizumab + azacitidine + epacadostat & ECHO 206 & NCT02959437 \\
\hline
\end{tabular}


Table 2 (Continued)

\begin{tabular}{|c|c|c|c|c|c|c|}
\hline Stage & $\begin{array}{l}\text { Specific } \\
\text { population }\end{array}$ & Phase & $\mathbf{n}$ & Experimental arm & Acronym & ClinicalTrials.gov \\
\hline Second line or more & & $1 / I 1$ & 197 & Pembrolizumab + AMG820 & & NCT027I 3529 \\
\hline Second line or more & & 1 & 18 & Pembrolizumab + LYC55716 & & NCT03396497 \\
\hline Second line or more & & 1 & 378 & Pembrolizumab + CPI006 + CPI444 & & NCT0345445I \\
\hline Second line or more & & II & 50 & $\begin{array}{l}\text { Pembrolizumab + recombinant EphB4-HSA } \\
\text { fusion protein }\end{array}$ & & NCT03049618 \\
\hline Second line or more & & 1 & 27 & Pembrolizumab + CM24 (MK60I8) & & NCT02346955 \\
\hline Second line or more & & 1 & 12 & Pembrolizumab + I-NMMA & & NCT03236935 \\
\hline Second line or more & & 1 & 22 & Pembrolizumab + GR-MD02 & & NCT02575404 \\
\hline Second line or more & & II & 74 & Pembrolizumab + acalabrutinib (ACPI96) & KEYNOTEI 66 & NCT02448303 \\
\hline Second line or more & & 1 & 15 & $\begin{array}{l}\text { Pembrolizumab + personalized cancer vaccine } \\
\text { (NEO-PVOI) + chemotherapy }\end{array}$ & & NCT0338087I \\
\hline Second line or more & & $1 / I 1$ & 40 & Pembrolizumab + idelalisib & & NCT03257722 \\
\hline Second line or more & & 1 & 57 & $\begin{array}{l}\text { Pembrolizumab + guadecitabine }+ \\
\text { mocetinostat (epigenetic drugs) }\end{array}$ & & NCT03220477 \\
\hline Second line or more & & I & 35 & Pembrolizumab + guadecitabine & HyPeR & NCT02998567 \\
\hline Second line or more & & II & 100 & Pembrolizumab + CC486 & & NCT02546986 \\
\hline Second line or more & & 1 & 155 & Pembrolizumab + ramucirumab & & NCT02443324 \\
\hline Second line or more & & 1 & 27 & Pembrolizumab + trigriluzole & & NCT03229278 \\
\hline Second line or more & & I & 6 & Pembrolizumab + lenvatinib & & NCT03006887 \\
\hline Second line or more & & 1 & 19 & Pembrolizumab + vaccine therapy & & NCT02432963 \\
\hline Second line or more & & 1 & 75 & Pembrolizumab + abemaciclib (LY2835219) & & NCT0277975I \\
\hline Second line or more & & $\mathrm{I} / \mathrm{II}$ & 67 & $\begin{array}{l}\text { Carboplatin or nab-paclitaxel + } \\
\text { pembrolizumab }\end{array}$ & & NCT02382406 \\
\hline Second line or more & Brain metastases & II & 64 & Pembrolizumab & & NCT02085070 \\
\hline Second line or more & Brain metastases & II & 53 & Pembrolizumab + bevacizumab & & NCT0268I549 \\
\hline Second line or more & Brain metastases & I & 30 & SBRT + pembrolizumab & & NCT02858869 \\
\hline Second line or more & Oligometastatic & II & 42 & Pembrolizumab after curative-intent treatment & & NCT02316002 \\
\hline $\begin{array}{l}\text { Post-erlotinib } \\
\text { progression }\end{array}$ & EGFR mutation & 1 & 38 & Pembrolizumab + afatinib & & NCT02364609 \\
\hline First line & ALK mutation & 1 & 70 & Crizotinib + pembrolizumab & KEYNOTE 050 & NCT025III 84 \\
\hline Second line or more & $\begin{array}{l}\text { EGFR/ALK } \\
\text { mutation }\end{array}$ & II & 62 & $\begin{array}{l}\text { Pembrolizumab + platinum-based doublet } \\
\text { chemotherapy }\end{array}$ & & NCT032429I5 \\
\hline Second line or more & KRAS mutation & I & 42 & Pembrolizumab + trametinib & & NCT03299088 \\
\hline Second line or more & Elderly & II & 82 & Pembrolizumab & & NCT03293680 \\
\hline Second line or more & $\mathrm{PS}=2$ & II & 60 & Pembrolizumab & PePS2 & NCT02733I59 \\
\hline Second line or more & HIV + patient & 1 & 39 & Pembrolizumab & & NCT02595866 \\
\hline \multicolumn{7}{|l|}{ Radiotherapy } \\
\hline & & II & 48 & SBRT + pembrolizumab & & NCT02658097 \\
\hline & & 1 & 32 & SBRT + pembrolizumab & SABRseq & NCT03307759 \\
\hline & & I/II & 104 & SBRT + pembrolizumab & & NCT0244474I \\
\hline & & $\mathrm{I} / \mathrm{II}$ & 60 & SBRT + pembrolizumab & & NCT02407I7I \\
\hline & & 1 & 24 & SBRT + pembrolizumab & PRIMING & NCT03368222 \\
\hline & & 1 & 35 & SBRT + pembrolizumab & & NCT02608385 \\
\hline & & II & 57 & $\begin{array}{l}\text { SBRT + oncolytic virus therapy before } \\
\text { pembrolizumab }\end{array}$ & STOMP & NCT03004I83 \\
\hline & & II & 40 & SRT + pembrolizumab & & NCT032I707I \\
\hline
\end{tabular}

Note: Major ongoing studies in bold font.

Abbreviations: SBRT, stereotactic body radiation therapy; PS, performance status; SRT, stereotactic radiation therapy. 
The randomized, open, Phase III KEYNOTE-042 trial was planned to include 1,240 patients in comparing firstline pembrolizumab vs chemotherapy for patients with advanced NSCLC with $>1 \%$ PDL1 expression. Patients were randomized at a 1:1 ratio between pembrolizumab $200 \mathrm{mg}$ Q3W for 2 years or until disease progression, with chemotherapy chosen by the physician. The results of this Phase III trial were presented in a presidential session at ASCO $2018 .^{38}$ The primary objective of the study was OS of patients with PDL1 $\geq 50 \%, \geq 20 \%$, and $\geq 1 \%$, with a statistically hierarchical analysis starting with patients with PDL1 $\geq 50 \%$. A total of 1,274 patients were included (637 in each arm), including 599 PDL1 patients $\geq 50 \%$ (47\%) and 818 PDL1 patients $\geq 20 \%$. The median follow-up at the time of analysis was 12.8 months. OS was significantly increased in the pembrolizumab arm for patients with PDL1 $>1 \%$ (16.7 vs 12.1 months, HR $0.81,95 \%$ CI $0.71-0.93 ; P=0.0018)$, PDL $1 \geq 20 \%$ (17.7 vs 13.0 months, HR $0.77,95 \%$ CI 0.64 $0.92 ; P=0.0020$ ), and PDL1 $\geq 50 \%$ (20.0 vs 12.2 months, HR $0.69,95 \%$ CI $0.56-0.84 ; P=0.0003$ ) compared to chemotherapy. OS gain was 4.5 months, 4.7 months, and 7.8 months for patients with PDL $1 \geq 1 \%$, PDL $1 \geq 20 \%$, and PDL1 $\geq 50 \%$ respectively. OS benefit was essentially driven by PDL $1>50 \%$ patients. For patients with PDL1 of $1 \%-49 \%$, an exploratory analysis found no difference in OS between pembrolizumab and chemotherapy (HR 0.92, $95 \%$ CI $0.77-1.11$ ). It should be noted that only $19.3 \%$ of patients on the chemotherapy arm benefited from a crossover to second-line immunotherapy. The toxicity profile was acceptable, with less grade $\geq 3$ toxicity in the pembrolizumab $\operatorname{arm}(17.8 \%$ vs $41 \%){ }^{38}$

For patients with advanced squamous-cell NSCLC, the randomized, double-blind, Phase III KEYNOTE-407 trial was designed to compare the efficacy and safety of a firstline pembrolizumab-platinum chemotherapy combination, regardless of PDL1 expression. The primary end point was PFS, with OS and PFS secondary-outcome criteria for the population with PDL1 $\geq 1 \%$ expression, ORR, DOR, and safety. The study recruited 560 patients, randomized 2:1 between pembrolizumab $200 \mathrm{mg}$ IV QW3 carboplatin-nabpaclitaxel and chemotherapy alone. Results of the second interim analysis were presented at ASCO 2018 by Paz-Ares et al, ${ }^{39}$ and are summarized in Table 1. OS increased significantly in the pembrolizumab + chemotherapy arm (median 15.9 months vs 11.3 months, HR $0.64,95 \%$ CI $0.49-0.85$; $P=0.0008)$. Similarly, median PFS increased from 4.8 months to 6.4 months (HR $0.56,95 \%$ CI $0.45-0.70 ; P<0.001$ ). ORR increased with pembrolizumab (57.9\% vs $38.4 \%)$. These positive results were observed regardless of the level of PDL1 expression. Tolerance was identical in both arms, with the same rate of grade 3-5 side effects (one treatment-related death in each arm) and of course more autoimmune events in the pembrolizumab arm $(28.8 \%$ vs $8.6 \%){ }^{39}$

For patients with advanced all-histology PDL1 $\geq 50 \%$ NSCLC, a randomized Phase-III trial with 542 planned inclusions compared fixed-dose $200 \mathrm{mg}$ pembrolizumab and a combination pembrolizumab fixed-dose $200 \mathrm{mg}$ Q3W-ipilimumab $1 \mathrm{mg} / \mathrm{kg}$ Q6W. The auxiliary PEARLS (KEYNOTE-091) trial, enrolling 1,380 patients, compared in a Phase III setting pembrolizumab (200 mg IV Q3W for 1 year) vs placebo in stage IB/IIIA NSCLC patients who had undergone surgical resections (lobectomy or pneumonectomy), with adjuvant chemotherapy or without. The principal outcome measure was disease-free survival. In the neoadjuvant setting, with the objective of improving disease-free survival and OS, the Phase-III KEYNOTE-671 trial, with 789 planned inclusions, evaluated the safety of pembrolizumab in combination with cisplatin-pemetrexed or cisplatin-gemcitabine chemotherapy depending on tumor histology before surgery, followed postoperatively by pembrolizumab alone in subjects with resectable stage IIB/IIIA NSCLC.

During ASCO 2018, Durm et al reported data from LUN 14-719 (Phase II). ${ }^{40}$ The objective of the trial was to evaluate the efficacy of consolidation therapy with pembrolizumab $200 \mathrm{mg}$ Q3W for 1 year in patients with unprogressive stage III NSCLC following concomitant chemoradiotherapy. The primary end point was time to metastatic disease or death. A total of 93 patients were included (92 evaluable): $59.8 \%$ stage IIIA N2 and $40.2 \%$ stage IIIB. The most commonly used chemotherapy regimen was carboplatin-paclitaxel ( $71.7 \%$ of cases). The median number of pembrolizumab cycles was 13.5 (1-19); 84\% of patients received at least four cycles and $43.5 \%$ of patients achieved overall pembrolizumab injections (one year of treatment). The median time to metastatic disease or death was 22.4 months (95\% CI 17.9-NR) and median PFS was 15.4 months (95\% CI 11.9-NR), and 16 patients (17.2\%) developed grade $\geq 2$ pneumonia ( $10.8 \%$ grade $2 \%$ and $5.4 \%$ grade $3 / 4$ ). A death related to radiation pneumonitis was observed. These results are quite consistent with those observed in the PACIFIC trial in terms of both tolerance and efficacy, whereas the beginning of immunotherapy was later (between 28 and 56 days after the end of radiotherapy-chemotherapy at 1-42 days in the PACIFIC trial). ${ }^{40}$

Data on other combinations are more limited. Results have been reported for the pembrolizumab-entinostat combination. Entinostat is a histone-deacetylase inhibitor with the potential to modulate myeloid-derived suppressor-cell 
functions, and may synergize with PD1/PDL1 inhibition. The preliminary results of a Phase II trial (57 patients) on entinostat plus pembrolizumab for patients with NSCLC previously treated with ICIs were reported at ASCO 2018. ${ }^{41}$ Five of the patients included achieved a confirmed partial response (ORR 9\%, 95\% CI 2.9-19.3). Among the responders, four had PDL1 expression $<1 \%$ in tissue collected at inclusion. Median DOR was 4.2 months. Twenty (35\%) patients experienced grade 3/4-related AEs, and six (10.5\%) experienced grade 3/4 IRAEs (three pneumonitides).

Thirty patients were given vorinostat, another histonedeacetylase inhibitor, and pembrolizumab in a Phase I/II trial. PDL 1 expression was $\geq 1 \%$ in 18 of $30(60 \%)$ and $\geq 50 \%$ in eleven of $30(37 \%)$ patients. Six patients were ICI-naïve and 24 ICI-pretreated. The disease-control rate was $67 \%$ for the entire study population. Among the ICI-pretreated patients, two achieved a partial response and ten had stable disease, and their PFS was 3.2 months compared to 7.6 months for ICI naïve patients. ${ }^{42}$ Pembrolizumab was evaluated in previously untreated patients with EGFR ${ }^{+} \operatorname{PDL}^{+}(>1 \%)$ NSCLC. Enrollment was stopped due to lack of efficacy after eleven of the 25 planned patients had been treated. Only one patient had an OR (ORR 9\%), but repeat analysis of this patient's tumor definitively proved the original report of an EGFR mutation to be erroneous.

\section{Current recommendations for ICls to treat NSCLC}

Regulatory agencies ${ }^{8-12,43,44}$ have now approved three ICIs to treat NSCLC - nivolumab, pembrolizumab, and atezolizumab - which are indicated for second-line or more therapy, ${ }^{6,13}$ regardless of PDL1-expression rate for nivolumab and atezolizumab, and restricted to $>1 \%$ expression for pembrolizumab. Two Phase III trials have evaluated nivolumab vs docetaxel, one for advanced squamous-cell NSCLC, the other for nonsquamous-cell NSCLC. ${ }^{8,44}$ In the latter Phase III trial on nivolumab vs docetaxel for patients whose non-squamous NSCLC had progressed after firstline chemotherapy, OS was prolonged (12.2 vs 9.4 months, respectively) without PFS improvement, but with better ORR for the nivolumab arm (19\% vs 12\%). Tumor PDL1 expression (Dako antibody clone 28-8) predicted a better response. OS for patients with $\geq 1 \%$ or $<1 \%$ PDL1 expression, respectively, lasted 17.7 vs 9.0 months or 10.5 vs 10.1 months comparing nivolumab vs second-line chemotherapy for each level. ${ }^{44}$

For atezolizumab, approval was based on the Phase III OAK trial. ${ }^{45}$ In that study, atezolizumab reduced the risk of death by $26 \%$ compared with docetaxel for patients with advanced NSCLC following the failure of platinum-based chemotherapy. Median OS was prolonged by 4.2 months. A survival benefit with atezolizumab was observed independently of PDL1 status or histology. For the intent-to-treat population, median OS was 13.8 months for atezolizumab vs 9.6 months for the docetaxel arm (HR 0.74, 95\% CI $0.63-0.87 ; P=0.0004)$. Among the $\mathrm{PDL}^{+}$group, median OS was 15.7 months for atezolizumab vs 10.3 months for the control arm (HR 0.74, 95\% CI 0.58-0.93; $P=0.0102$ ). Among PDL1 ${ }^{-}$patients, median OS was 12.6 months for atezolizumab vs 8.9 months for the docetaxel group (HR $0.75,95 \%$ CI $0.59-0.96 ; P=0.0205){ }^{45}$

First-line pembrolizumab monotherapy has been approved only for stage IIIB/IV NSCLC with $\geq 50 \%$ PDL1 expression and without EGFR mutation, or $A L K$ or $R O S 1$ (transmembrane tyrosine-kinase receptor) rearrangement. ${ }^{46}$ Moreover, in January 2017, based on the promising KEYNOTE-021 trial results, the FDA approved first-line pembrolizumab in combination with carboplatin-pemetrexed chemotherapy for patients with nonsquamous NSCLC independently of PDL1 expression. ${ }^{12}$ In February 2018, the FDA approved durvalumab, a human $\left(\operatorname{IgG}_{1 \mathrm{~K}}\right)$ monoclonal antibody that blocks PDL1 interaction with PD1 and CD80, to treat stage III, unresectable, locally advanced NSCLC that had not progressed after concurrent chemoradiotherapy, based on the Phase III PACIFIC trial results showing that durvalumab prolonged PFS by 11.2 months compared to placebo (16.8 vs 5.6 months, HR $0.52,95 \%$ CI $0.42-0.65 ; P<0.0001)$. Their respective PFS rates in favor of durvalumab were $55.9 \%$ vs $35.3 \%$ at 12 months and $44.2 \%$ vs $27.0 \%$ at 18 months. ${ }^{43}$

\section{PDLI expression: the good biomarker?}

Unlike other ICIs (eg, nivolumab or atezolizumab), pembrolizumab monotherapy depends on tumor-cell PDL1 expression ( $\geq 1 \%$ for second-line, $\geq 50 \%$ for first line), which is frequent in NSCLC. ${ }^{26,47,48}$ Selecting patients based on their PDL1-expression level obtains better pembrolizumab efficacy, especially for first-line therapy. It is essential to identify biomarkers able to select potential responders to immunotherapy more optimally.

The results of several studies have shown that ICI efficacy was associated with tumor-cell PDL1-expression level. ${ }^{1744,47,49-52}$ Indeed, in the KEYNOTE-001 trial, ${ }^{28}$ a significant association was found between PDL1 expression and pembrolizumab efficacy, with an optimal expression threshold of $50 \%$ according to receiver-operating-characteristic 
curve analysis. Tumors with $\geq 50 \%$ PDL1 expression were defined as strongly positive, those with $1 \%-49 \%$ expression as weakly positive, and $<1 \%$ expression as negative. ${ }^{28}$ For the validation cohort (KEYNOTE-001), with 313 patients according to the three aforementioned PDL1 groups, ORRs were $45.2 \%, 16.5 \%$, and $10.7 \%$ and median PFS lasted 6.3 , 4.1 , and 4.0 months, respectively. ${ }^{28}$

For nivolumab, in the Phase I trial that included all types of solid tumors, including advanced NSCLC, only PDL1 ${ }^{+}$ ( $\geq 5 \%$ ) tumors responded. ${ }^{53}$ The results of the Check-Mate 057 trial found better nivolumab efficacy in patients with strongly expressed PDL1. ${ }^{44}$ The Phase I atezolizumab trial and OAK study also showed an association between PDL1 levels expressed by intratumor-infiltrating immune cells and clinical response. ${ }^{44,54} \mathrm{~A}$ meta-analysis of seven studies on ICI-treated NSCLC patients $(n=914)$ found that those with $\mathrm{PDL}^{+}(\geq 1 \%)$ tumors had ORRs significantly higher than patients whose tumors were PDL1- ${ }^{-}$(OR 2.44, 95\% CI $1.61-3.68){ }^{48}$

Nonetheless, clinical responses have been observed in patients with PDL1 ${ }^{-}$tumors, showing that IHC-determined PDL1 expression is an imperfect marker predictive of response to ICIs. ${ }^{8-10,44,55}$ Several things could explain those findings. In the first studies, IHC was not standardized, and several labeling techniques were used with at least four monoclonal antibodies (clones 22C3, 28-8, SP142, and SP263) and developed as companion tests for different PD1 or PDL1 inhibitors. Several groups decided to compare the four available antibodies on different platforms. Hirsch et al showed that three (28-8, 22C3, and SP263) of the four reagents were indeed comparable in terms of sensitivity, specificity, and reproducibility. ${ }^{56}$ Adam et al found that antibodies 28-8, $22 \mathrm{C} 3$, and SP263 had similar analytical performances for labeling tumor cells in seven different centers. ${ }^{57}$

PDL1 expression is a qualitative variable, which explains the choices of different positivity thresholds depending on the study. ${ }^{10,11,43}$ According to a recent meta-analysis of antiPD1/PDL1 therapeutic agents, a 5\% threshold seemed to have maximum discriminatory power (OR 2.72, $P=0.01) .{ }^{52}$ In addition, heterogeneous PDL1 expression is possible in a given tumor or between the primary tumor and its metastases. ${ }^{58}$

Finally, in most studies, PDL1 expression by NSCLC cells was assessed by IHC, without taking into account the immune microenvironment, but no definitive information is available on the role of $\mathrm{PDL}^{+}$macrophages in the prediction of response to ICIs. ${ }^{49,50}$ Moreover, no consensus exists about the dynamic character or not of PDL1 expression over time.
Indeed, PDL1 can be induced, notably by IFN ${ }_{\gamma}{ }^{18}$ and tumors not expressing that marker can become PDL1 ${ }^{+}$because of a chronic inflammatory phenomenon. ${ }^{18}$ Because modification of PDL1-expression rate has also been observed during NSCLC treatment, interest in rebiopsy of tumors before starting anti-PD1/PDL1 therapy has been raised. ${ }^{59}$

Other potential biomarkers for the selection of populations sensitive to ICIs have emerged in the literature, eg, the tumor-mutation burden (TMB). Because the most effective ICIs against tumors have elevated levels of somatic mutations, it has been suggested that TMB could play an important role in the response to PD1/PDL1 inhibitors. ${ }^{50}$ Sequencing of the entire exome of pembrolizumab-treated NSCLC showed that a high TMB was associated with a PFS benefit and higher ORR ${ }^{60} \mathrm{~A}$ study evaluating the role of comprehensive cancergene panels (300 genes) to estimate TMB showed that the association between TMB and ICI clinical benefit was also seen when cancer-gene panels were used to estimate TMB..$^{51}$ Furthermore, the results of several studies have shown that TMB was not associated with PDL1 expression. ${ }^{50}$ The use of PDL1 expression and TMB might obtain a higher predictive value for response to ICIs than individual use of one or the other of these biomarkers. ${ }^{61}$

\section{Discussion}

The KEYNOTE-024 trial results showed the superiority of first-line immunotherapy over platinum-based chemotherapy for advanced PDL1 $\geq 50 \%$ NSCLCs. ${ }^{11}$ Pembrolizumab obtained the most benefit against squamous-cell carcinoma in terms of PFS, with HR of 0.35 (95\% CI $0.17-0.71)$. That was a major breakthrough for these tumors, for which no therapeutic progress had been made for several years. With regard to OS benefit, it has to be considered that only $50 \%$ of the chemotherapy-arm patients benefited from crossover to pembrolizumab at disease progression. The safety profile was quite acceptable for a first-line treatment, requiring monitoring and providing information on IRAEs. Despite reservations about PDL1 expression by tumor cells addressed herein, this biomarker enables selection of patients getting the most benefits from first-line pembrolizumab. In all the international recommendations, pembrolizumab is the standard of care for patients with advanced PDL1 $\geq 50 \%$ NSCLC without EGFR mutation or $A L K$ rearrangement and PS 0-1.

For patients whose tumors express $<50 \%$ PDL1, data on the pembrolizumab-chemotherapy combination seem promising. The results for KEYNOTE-021 cohort $\mathrm{G}$ showed doubling of the ORR (55\% vs $28 \%$ ), and the recent findings of the KEYNOTE-189 trial confirmed the 
superiority of the ICI-chemotherapy combination compared to chemotherapy alone, regardless of PDL1 status. ${ }^{12,47}$ It should be underlined that the magnitude of the pembrolizumab PFS benefit (alone or with chemotherapy) compared to chemotherapy alone was the same in the three trials: 0.53 in KEYNOTE-021, 0.52 in KEYNOTE-189, and 0.50 in KEYNOTE-024. ${ }^{11,12,36}$ The safety of the pembrolizumabpemetrexed-platinum combination was very close to that of chemotherapy alone. ${ }^{36}$ KEYNOTE-189 trial results should in the near future lead to discussion of that combination as the new standard of care for patients whose NSCLC cells express $\geq 50 \%$ PDL 1 .

The challenge over the next 2 years is to identify predictive biomarkers of ICI efficacy. PDL1 expression is not optimal for the selection of patients to receive ICIs. Many patients have NSCLC not expressing PDL1, and others have hyperprogression under ICIs, despite having PDL1+ tumors. The search for the best combination of predictive biomarkers (PDL1 ${ }^{+}, \mathrm{TMB}$, and/or others) must be a top-priority objective for research teams. Simplification and harmonization of techniques for the reproducibility of biomarker detection are other prominent foci of interest for the near future. Academic societies and regulatory authorities should encourage and launch major projects to harmonize practices and lower the costs of testing for these biomarkers.

\section{Conclusion}

The advent of ICIs, notably pembrolizumab, has prolonged NSCLC patient survival. The efficacy of second-line pembrolizumab monotherapy has been shown in patients with tumors with $\geq 1 \%$ PDL1 expression and as first-line treatment for patients with $\geq 50 \%$ of tumor cells expressing PDL1, and more recently as first-line therapy in combination with a pemetrexed-platinum-based chemotherapy for advanced NSCLC, regardless of PDL1 status. At present, PDL1 expression is the only predictive biomarker validated for the selection of patients who could benefit from pembrolizumab. It is not perfect, and in future TMB could help improve patient selection.

\section{Author contributions}

All authors contributed to data analysis, drafting and revising the paper and agreeing to be accountable for all aspects of the work. All authors read and approved the final manuscript.

\section{Disclosure}

OB has served as a consultant for AstraZeneca, Roche, and MSD. GRB served as a consultant for Roche. In the past
5 years, $\mathrm{CC}$ has received fees for attending scientific meetings, speaking, organizing research, or consulting from AstraZeneca, Boehringer Ingelheim, GSK, Roche, Sanofi Aventis, BMS, MSD, Lilly, Novartis, and Amgen. The authors report no other conflicts of interest in this work.

\section{References}

1. Ferlay J, Soerjomataram I, Dikshit R, et al. Cancer incidence and mortality worldwide: sources, methods and major patterns in GLOBOCAN 2012. Int J Cancer. 2015;136(5):E359-E386.

2. Jemal A, Siegel R, Xu J, Ward E. Cancer statistics, 2010. CA Cancer J Clin. 2010;60(5):277-300.

3. Arnold M, Pandeya N, Byrnes G, et al. Global burden of cancer attributable to high body-mass index in 2012: a population-based study. Lancet Oncol. 2015;16(1):36-46.

4. Masters GA, Temin S, Azzoli CG, et al. Systemic therapy for stage IV non-small-cell lung cancer: American Society of Clinical Oncology clinical practice guideline update. J Clin Oncol. 2015;33(30):3488-3515.

5. Besse B, Adjei A, Baas P, et al. 2nd ESMO Consensus Conference on Lung Cancer: non-small-cell lung cancer first-line/second and further lines of treatment in advanced disease. Ann Oncol. 2014;25(8): $1475-1484$.

6. Ettinger DS, Wood DE, Akerley W, et al. NCCN guidelines insights: nonsmall cell lung cancer, version 4.2016. J Natl Compr Canc Netw. 2016; 14(3):255-264.

7. Hanna N, Johnson D, Temin S, et al. Systemic therapy for stage IV nonsmall-cell lung cancer: American Society of Clinical Oncology clinical practice guideline update. J Clin Oncol. 2017;35(30):3484-3515.

8. Brahmer J, Reckamp KL, Baas P, et al. Nivolumab versus docetaxel in advanced squamous-cell non-small-cell lung cancer. NEngl J Med. 2015; 373(2):123-135.

9. Fehrenbacher L, Spira A, Ballinger M, et al. Atezolizumab versus docetaxel for patients with previously treated non-small-cell lung cancer (POPLAR): a multicentre, open-label, phase 2 randomised controlled trial. Lancet. 2016;387(10030):1837-1846.

10. Herbst RS, Baas P, Kim DW, et al. Pembrolizumab versus docetaxel for previously treated, PD-L1-positive, advanced non-small-cell lung cancer (KEYNOTE-010): a randomised controlled trial. Lancet. 2016;387(10027):1540-1550.

11. Reck M, Rodríguez-Abreu D, Robinson AG, et al. Pembrolizumab versus chemotherapy for PD-L1-positive non-small-cell lung cancer. N Engl J Med. 2016;375(19):1823-1833.

12. Langer CJ, Gadgeel SM, Borghaei H, et al. Carboplatin and pemetrexed with or without pembrolizumab for advanced, non-squamous nonsmall-cell lung cancer: a randomised, phase 2 cohort of the open-label KEYNOTE-021 study. Lancet Oncol. 2016;17(11):1497-1508.

13. Novello S, Barlesi F, Califano R, et al. Metastatic non-small-cell lung cancer: ESMO clinical practice guidelines for diagnosis, treatment and follow-up. Ann Oncol. 2016;27 Suppl 5:v1-v27.

14. Finn OJ. Immuno-oncology: understanding the function and dysfunction of the immune system in cancer. Ann Oncol. 2012;23 Suppl 8: viii6-viii9.

15. Finn OJ. A believer's overview of cancer immunosurveillance and immunotherapy. J Immunol. 2018;200(2):385-391.

16. Armand P. Immune checkpoint blockade in hematologic malignancies. Blood. 2015;125(22):3393-3400.

17. Brahmer JR, Pardoll DM. Immune checkpoint inhibitors: making immunotherapy a reality for the treatment of lung cancer. Cancer Immunol Res. 2013;1(2):85-91.

18. Pardoll DM. The blockade of immune checkpoints in cancer immunotherapy. Nat Rev Cancer. 2012;12(4):252-264.

19. Agata Y, Kawasaki A, Nishimura H, et al. Expression of the PD-1 antigen on the surface of stimulated mouse $\mathrm{T}$ and B lymphocytes. Int Immunol. 1996;8(5):765-772. 
20. Blank C, Brown I, Peterson AC, et al. PD-L1/B7H-1 inhibits the effector phase of tumor rejection by $\mathrm{T}$ cell receptor (TCR) transgenic CD8+ T cells. Cancer Res. 2004;64(3):1140-1145.

21. Freeman GJ, Long AJ, Iwai Y, et al. Engagement of the PD-1 immunoinhibitory receptor by a novel B7 family member leads to negative regulation of lymphocyte activation. J Exp Med. 2000;192(7):1027-1034.

22. Dong H, Strome SE, Salomao DR, et al. Tumor-associated B7-H1 promotes T-cell apoptosis: a potential mechanism of immune evasion. Nat Med. 2002;8(8):793-800.

23. Keir ME, Butte MJ, Freeman GJ, Sharpe AH. PD-1 and its ligands in tolerance and immunity. Anпи Rev Immunol. 2008;26:677-704.

24. Garon EB. Current perspectives in immunotherapy for non-small cell lung cancer. Semin Oncol. 2015;42 Suppl 2:S11-S18.

25. Scarpace SL. Metastatic squamous cell non-small-cell lung cancer (NSCLC): disrupting the drug treatment paradigm with immunotherapies. Drugs Context. 2015;4:212289.

26. Alvarez JG, González-Cao M, Karachaliou N, et al. Advances in immunotherapy for treatment of lung cancer. Cancer Biol Med. 2015;12(3): 209-222.

27. Barone A, Hazarika M, Theoret MR, et al. FDA approval summary: pembrolizumab for the treatment of patients with unresectable or metastatic melanoma. Clin Cancer Res. 2017;23(19):5661-5665.

28. Garon EB, Rizvi NA, Hui R, et al. Pembrolizumab for the treatment of non-small-cell lung cancer. N Engl J Med. 2015;372(21):2018-2028.

29. Gandhi L, Balmanoukian A, Hui R, et al. Abstract CT105: MK-3475 (anti-PD-1 monoclonal antibody) for non-small cell lung cancer (NSCLC): antitumor activity and association with tumor PD-L1 expression. Cancer Res. 2014;74(19 Supplement):CT105.

30. Hui R, Garon EB, Goldman JW, et al. Pembrolizumab as first-line therapy for patients with PD-L1-positive advanced non-small cell lung cancer: a phase 1 trial. Ann Oncol. 2017;28(4):874-881.

31. Lisberg A, Tucker DA, Goldman JW, et al. Treatment-related adverse events predict improved clinical outcome in NSCLC patients on KEYNOTE-001 at a single center. Cancer Immunol Res. 2018;6(3):288-294.

32. Brahmer J, Rodríguez-Abreu D, Robinson A, et al. Updated analysis of KEYNOTE-024: pembrolizumab vs platinum-based chemotherapy for advanced NSCLC with PD-L1 TPS $\geq 50 \%$. J Thorac Oncol. 2017;12(11):S1793-S1794.

33. Brahmer JR, Rodríguez-Abreu D, Robinson AG, et al. Health-related quality-of-life results for pembrolizumab versus chemotherapy in advanced, PD-L1-positive NSCLC (KEYNOTE-024): a multicentre, international, randomised, open-label phase 3 trial. Lancet Oncol. 2017;18(12):1600-1609.

34. Gadgeel SM, Stevenson J, Langer CJ, et al. Pembrolizumab (Pembro) plus chemotherapy as front-line therapy for advanced NSCLC: KEYNOTE-021 cohorts A-C. J Clin Oncol. 2016;34(15 Suppl):9016.

35. Gentzler R, Langer C, Borghaei C, et al. 24-Month overall survival from KEYNOTE-021 cohort G: pemetrexed-carboplatin plus pembrolizumab as first-line therapy for advanced nonsquamous NSCLC. J Clin Oncol. 2018;36 Suppl:9026.

36. Gandhi L, Rodríguez-Abreu D, Gadgeel S, et al. Pembrolizumab plus chemotherapy in metastatic non-small-cell lung cancer. $N$ Engl J Med 2018;378(22):2078-2092.

37. Gubens MA, Sequist LV, Stevenson J, et al. Phase I/II study of pembrolizumab (pembro) plus ipilimumab (ipi) as second-line therapy for NSCLC: KEYNOTE-021 cohorts D and H. J Clin Oncol. 2016; 34(15 Suppl):9027.

38. Lopes G, Wu YL, Kudaba I, et al. Pembrolizumab (pembro) versus platinum-based chemotherapy (chemo) as first-line therapy for advanced/metastatic NSCLC with a PD-L1 tumor proportion score (TPS) $\geq 1 \%$ : open-label, phase 3 KEYNOTE-042 study. J Clin Oncol. 2018;36(18 Suppl):LBA4

39. Paz-Ares L, Luft A, Tafreshi A, et al. Phase 3 study of carboplatinpaclitaxel/nab-paclitaxel (Chemo) with or without pembrolizumab (Pembro) for patients (Pts) with metastatic squamous (Sq) non-small cell lung cancer (NSCLC). J Clin Oncol. 2018;36 Suppl:105.
40. Durm G, Althouse S, Sadiq A, et al. Phase II trial of concurrent chemoradiation with consolidation pembrolizumab in patients with unresectable stage III non-small cell lung cancer: Hoosier Cancer Research Network LUN 14-179. J Clin Oncol. 2018;36 Suppl:8500.

41. Gandhi L, Janne P, Opyrchal M, et al. Efficacy and safety of entinostat (Ent) and pembrolizumab (Pembro) in patients with non-small cell lung cancer (NSCLC) previously treated with anti-PD-(L)1 therapy. J Clin Oncol. 2018;36 Suppl:9036.

42. Saltos A, Tanvetyanon T, Williams C, et al. Phase I/IB study of pembrolizumab and vorinostat in patients with metastatic NSCLC (mNSCLC). J Clin Oncol. 2018;36 Suppl:9046.

43. Antonia SJ, Villegas A, Daniel D, et al. Durvalumab after chemoradiotherapy in stage III non-small-cell lung cancer. $N$ Engl J Med. 2017;377(20):1919-1929.

44. Borghaei H, Paz-Ares L, Horn L, et al. Nivolumab versus docetaxel in advanced nonsquamous non-small-cell lung cancer. $N$ Engl $J$ Med. 2015;373(17):1627-1639.

45. Rittmeyer A, Barlesi F, Waterkamp D, et al. Atezolizumab versus docetaxel in patients with previously treated non-small-cell lung cancer $(\mathrm{OAK})$ : a phase 3, open-label, multicentre randomised controlled trial. Lancet. 2017;389(10066):255-265.

46. Sul J, Blumenthal GM, Jiang X, He K, Keegan P, Pazdur R. FDA approval summary: pembrolizumab for the treatment of patients with metastatic non-small cell lung cancer whose tumors express programmed death-ligand 1. Oncologist. 2016;21(5):643-650.

47. Lin Z, Xu Y, Zhang Y, et al. The prevalence and clinicopathological features of programmed death-ligand 1 (PD-L1) expression: a pooled analysis of literatures. Oncotarget. 2016;7(12):15033-15046.

48. Passiglia F, Bronte G, Bazan V, et al. PD-L1 expression as predictive biomarker in patients with NSCLC: a pooled analysis. Oncotarget. 2016;7(15):19738-19747.

49. Rizvi NA, Hellmann MD, Snyder A, et al. Mutational landscape determines sensitivity to PD-1 blockade in non-small cell lung cancer. Science. 2015;348(6230):124-128.

50. Rizvi H, Sanchez-Vega F, La K, et al. Molecular determinants of response to anti-programmed cell death (PD)-1 and anti-programmed death-ligand 1 (PD-L1) blockade in patients with non-small-cell lung cancer profiled with targeted next-generation sequencing. J Clin Oncol. 2018;36(7):633-641.

51. Xia B, Herbst RS. Immune checkpoint therapy for non-small-cell lung cancer: an update. Immunotherapy. 2016;8(3):279-298.

52. Khunger M, Rakshit S, Schalper KA, et al. Meta-analysis of tumor PD-L1 expression as a predictive biomarker of benefit from PD-1/ PD-L1 axis inhibitors in solid tumors. J Clin Oncol. 2016;34(15 Suppl): 11603.

53. Gettinger SN, Horn L, Gandhi L, et al. Overall survival and long-term safety of nivolumab (anti-programmed death 1 antibody, BMS-936558, ONO-4538) in patients with previously treated advanced non-small-cell lung cancer. J Clin Oncol. 2015;33(18):2004-2012.

54. Mizugaki H, Yamamoto N, Murakami H, et al. Phase I dose-finding study of monotherapy with atezolizumab, an engineered immunoglobulin monoclonal antibody targeting PD-L1, in Japanese patients with advanced solid tumors. Invest New Drugs. 2016;34(5):596-603.

55. Peters S, Kerr KM, Stahel R. PD-1 blockade in advanced NSCLC: a focus on pembrolizumab. Cancer Treat Rev. 2018;62:39-49.

56. Hirsch FR, Mcelhinny A, Stanforth D, et al. PD-L1 immunohistochemistry assays for lung cancer: results from phase 1 of the Blueprint PD-L1 IHC Assay Comparison Project. J Thorac Oncol. 2017;12(2): 208-222.

57. Adam J, le Stang N, Rouquette I, et al. Multicenter French harmonization study for PD-L1 IHC testing in non-small cell lung cancer. Ann Oncol. 2018;29(4):953-958.

58. Ilie M, Long-Mira E, Bence C, et al. Comparative study of the PD-L1 status between surgically resected specimens and matched biopsies of NSCLC patients reveal major discordances: a potential issue for antiPD-L1 therapeutic strategies. Ann Oncol. 2016;27(1):147-153. 
59. Giunchi F, Degiovanni A, Daddi N, et al. Fading with time of PD-L1 immunoreactivity in non-small cells lung cancer tissues: a methodological study. Appl Immunohistochem Mol Morphol. Epub 2016 Oct 31.

60. Campesato LF, Barroso-Sousa R, Jimenez L, et al. Comprehensive cancer-gene panels can be used to estimate mutational load and predict clinical benefit to PD-1 blockade in clinical practice. Oncotarget. 2015;6(33):34221-34227.
61. Hellmann MD, Ciuleanu TE, Pluzanski A, et al. Nivolumab plus ipilimumab in lung cancer with a high tumor mutational burden. $N$ Engl J Med. 2018;378(22):2093-2104

\section{Publish your work in this journal}

OncoTargets and Therapy is an international, peer-reviewed, open access journal focusing on the pathological basis of all cancers, potential targets for therapy and treatment protocols employed to improve the management of cancer patients. The journal also focuses on the impact of management programs and new therapeutic agents and protocols on

patient perspectives such as quality of life, adherence and satisfaction. The manuscript management system is completely online and includes a very quick and fair peer-review system, which is all easy to use. Visit http://www.dovepress.com/testimonials.php to read real quotes from published authors. 